\title{
Design of Bus Protocol Intelligent Initiation System Based On
}

\section{RS485}

\author{
Liming $\mathrm{Li}^{1, \mathrm{a}}$, Qiao $\mathrm{Lu}^{1, \mathrm{~b}}$ and Guofu Yin ${ }^{1, \mathrm{c}}$ \\ ${ }^{1}$ State Key Laboratory of Applied physic-Chemistry Research, China \\ abiq213@163.com, ${ }^{\mathrm{b}}$ chufengde01@163.com, cyinguofu521@126.com
}

\begin{abstract}
In order to design an effective and reliable RS485 bus protocol based on RS485 bus, this paper introduces the structure and transmission mode of the command frame and the response frame, and also introduce four control measures and the communication in order to process quality of this system. The communication protocol is open, tolerant, reliable and fast, and can realize ignition more reliable and accurate in the intelligent initiation system.
\end{abstract}

Keywords. Intelligent initiation system; RS485 bus; communication protocol; pyrotechnics.

\section{Introduction}

The increasing demand of ordnance initiation system in the volume, weight, low energy consumption and integrated circuit with low energy input, high performance output led to this new way of detonating ordnance come out, which called addressable integrated intelligent initiation system (Intelligent detonation system). As shown in Figure 1, compared to the traditional intelligent ignition system ignition system: First, Time division multiplexing detonating control bus instead of multiple high current wire, in the guidance and control of the input signal module instruction function, all the detonators on the bus can be controlled by the bus controller, and the bus topology determines the changes in the number of wire does not increase with the initiation of nodes, which is very conducive to multi section industrial products group detonated ignition, greatly reducing the number of wires; Second, insurance and firing circuit are integrated into smart initiating device from the ignition controller, that is greatly reduces the system's volume and weight; Third, semiconductor bridge replaced the traditional hot bridge wire device, greatly reduces demand of fire energy, which can strengthen not firing performance of the system.
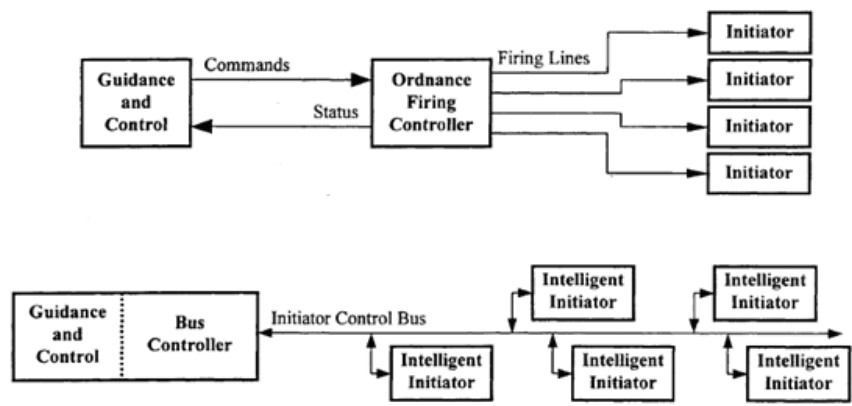

Fig. 1. Comparison between traditional and intelligent initiating system. 
The "WizordTM" intelligent initiating system is a typical example of the system, which can be not only applied to the large launch vehicle and tactical missile,but also extended to the steering propulsion and attitude control system. As shown in Figure 2, it includes a bus controller, a number of intelligent initiating devices, a bus containing four transmission lines, and 124 initiating devices, which can relieve security or ignition. Figure 3 shows the the photo of the bus controller, multi node cable and the initiator [3-4].



Fig. 2. "WizordTM" intelligent initiating system.

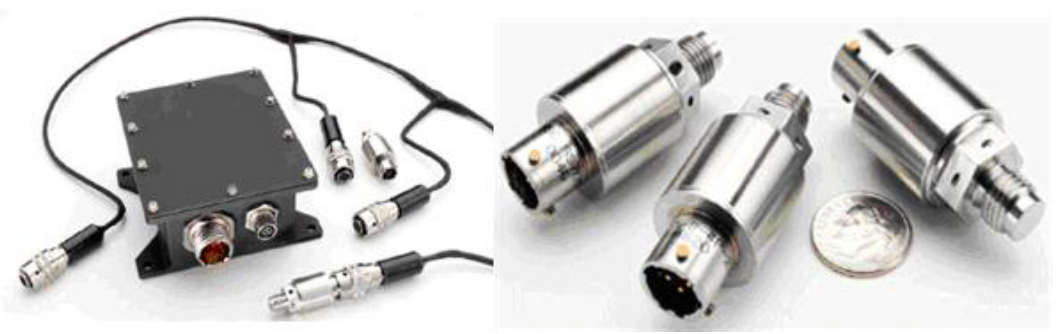

Fig. 3. "WizordTM" system hardware.

Intelligent detonation system, fundamentally changed the structure of initiation system, greatly optimizing the initiation system performance, reducing the cost of initiation system .Can be used for crew escape and carrier rocket, tactical missiles, aircraft ordnance initiation situations, realize multi-point synchronous or asynchronous accurate detonation or ignition $[1,3]$.

\section{Intelligent initiating system}

According to the the study of intelligent initiation system at home and abroad, a kind of intelligent initiation system is designed,which can realize multi point synchronous or asynchronous accurate initiation or ignition. As shown in Figure 4, it includes a PC machine, a bus controller, a single chip controller, a plurality of smart initiating devices, and a multi connector shielded twisted pair cable. The whole system is divided into the following four parts:

(1) $\mathrm{PC}$

PC simulation guidance and control module, send instructions to the intelligent initiator, and the implementation results of each instruction is displayed on the user interface,which is easy to understand the process of the system.

(2) Bus controller

The bus controller receives the energy and instructions from the $\mathrm{PC}$, and through the control of the energy switch or the firing energy switch, the energy and the instruction is delivered to the designated initiator.

(3) Intelligent initiating device

In the intelligent initiating device, the system is integrated with the relieve security and ignition circuit, security and firing switch controlled by the received instruction, firing through capacitor discharge. In addition to the solution of the security and firing instructions, but also the implementation of state detection, write address and so on.

(4) Initiation control bus 
The initiating control bus is composed of an operating power line, an insurance energy line, a communication line and a ground wire. Among them, the communication bus adopts RS485 standard, which can achieve high efficiency, accurate and reliable communication. It is still able to communicate with the transmission distance and the interference of the more serious, and meet the requirements of multi point initiation and small scale integration, which allows access to up to 256 initiator $[5,6]$.

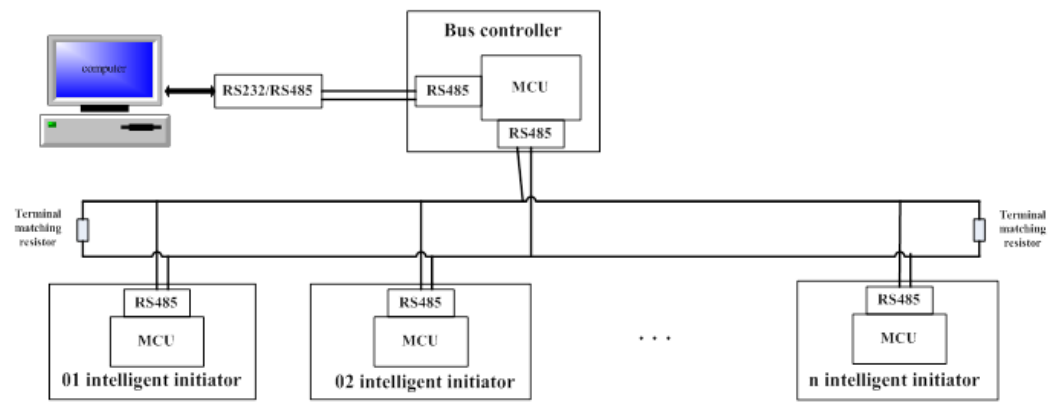

Fig.4. Schematic diagram of the intelligent initiating system constructed by RS485.

This is a master slave network that allows a master to communicate with one or more slave machine, the slave machine (initiator) can not send data, everything is controlled by the master (bus controller). Using the command / response mode, the bus controller forwards the instruction from the PC to the address of the initiator, and the initiator will perform the results to answer the bus controller in the way of the response frame.This way can ensure the system is reliable communication .Bus controller sends 2 types of requests to the initiator: (1)a single address mode: the master addresses a separate slave, which is received and processed from the machine, and returns an answer to the master. Every address from the machine must be unique. (2)broadcast mode: the master sends a request to all slave machines, every slave machine receive and process the request. After the provision delay time, each slave machine return the response to the master machine. By adopting the method of time response, it is effective to avoid the bus conflicts caused by multiple machines from the same time to the bus. All from the machine to share a broadcast address [7, 8].

\section{RS485 bus}

RS485 as the communication bus of intelligent initiating system, has the following advantages:

(1) According to the different input impedance of the receiver, the RS485 bus can be hung from 32 nodes, 64 nodes, 128 nodes, 256 nodes, is conducive to achieve more than one point of initiation.

(2) RS485 is used to transmit and receive the shield twisted pair, and the anti - mode interference capability is strong.

(3) RS485 bus structure is simple (half duplex 2 communication lines, full duplex 4 communication lines), the drive chip is small, and it is beneficial for the integration of the system.

(4) The sensitivity of the RS485 bus transceiver is high, and can detect the voltage of $200 \mathrm{mV}$, and the transmission signal can be recovered from the $\mathrm{Km}$ distance.

Due to the high reliability requirements of the initiating system, we must pay attention to the use of RS485 bus:

(1)In order to match the communication line impedance, RS485 bus terminal need to access two terminating resistance, the resistance is equal to the characteristic impedance of the cable transmission. The characteristic impedance of the twisted pair is generally $100 \Omega$ to $150 \Omega$, which is related to the thickness of the insulation material and the diameter of the wire. The best matched impedance of the system should be measured by experiment.

(2)The maximum transmission rate of RS485 is $10 \mathrm{Mb} / \mathrm{s}$, the transmission distance is only $10 \mathrm{~m}$ at this rate, the most distant transmission distance is about 1219 meters, the transmission rate is only $105 \mathrm{~b} / \mathrm{s}$. The length of twisted pair is in inverse proportion to transmission rate, and the maximum transmission rate of $100 \mathrm{~m}$ long twisted pair is $1 \mathrm{Mb} / \mathrm{s}$. In 
order to achieve the best transmission rate under different transmission distance, the limit transmission rate of each range can be measured by a large number of experiments.

(3)The length of the lead wire from the bus to each node should be as short as possible in order to minimize the impact of the reflected signal from the lead wire to the bus signal $[5,6]$.

\section{Bus protocol design}

RS485 bus only provides the electrical standard, in order to ensure the system communication is accurate and reliable , a clear provision have to be made on the data transmission mode, synchronization control mode (frame synchronization), error handling and other issues, in order to develop a general purpose of the intelligent initiation system of communication protocol.

In order to meet the need of multi point synchronization and different transmission distance of the intelligent initiating system, the communication protocol has two characteristics:

Baud rate selectable: the system is no longer limited to a particular baud rate, because the bandwidth resources can not be efficient used. According to different transmission distance, the best transmission rate is selected.

The broadcast instruction may be responsive: the slave machine can only perform the instruction but not to make any response. The time-sharing return mode, each slave machine can response, and if there is a slave machine dose not respond,master command retransmission, thus ensuring the synchronization and reliability of radio communication.

\section{1 data frame structure design}

Data frame is the basic unit of information transfer in the single chip computer system. According to the function, the data frame is divided into the command frame and the response frame, as shown in Figure 5.

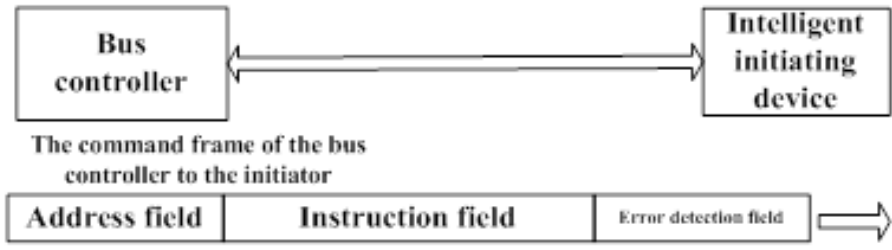

Reply frame to the bus controller

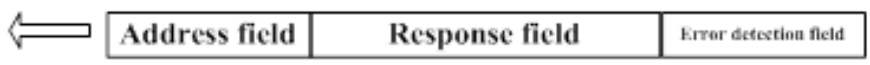

Fig. 5. Command frame and response frame.

The data frame structure is given below:

Table 1 Data frame structure.

\begin{tabular}{|c|c|c|l|c|}
\hline Start byte & Address byte & Function byte & Data byte & Check byte \\
\hline$*$ & 1 byte & 1 byte & none, 1 byte, 2 byte & 1 byte \\
\hline
\end{tabular}

- Start byte: the beginning of the identification of a frame of data, set to "*" characters, the value is $0 \times 2 \mathrm{~A}$.

- Address byte: in the case of an intelligent initiating system, the address of the initiator is indicated. $0 \mathrm{x} 00$ - $0 \mathrm{xEF}$, respectively, on behalf of the $0-254$ initiator, the unique address, 0xFF as a general broadcast address; in the handheld detection and the encoder and the initiator, the byte is fixed to $0 \mathrm{xFF}$.

- Function byte: the low 4 bits are the command code range. The effective command code is $0 \mathrm{x} 07$ - 0x01, which is 7 kinds of commands, such as address, state detection, system self check, single address charging, charging, single access, broadcast, etc.. 
- Data byte: additional information about the command code. Write address command requires 1 address byte, single access, broadcast firing command to 2 time delay time, other commands no data byte.

- Check and byte: binary arithmetic and the.

- Each byte of the transmission process contains 8 bit binary code, plus a start bit "0", and a stop bit "1", and a total of 10 bit [7-8].

\section{2 measures to improve the quality of communication}

(1)The frame structure is simple: the data frame only contains valid data and necessary expenses, which can improve the transmission rate, and reduce the bit error rate.

(2)Error control: Communication between the two parties using data and verification, frame check speed, simple process, although not error correction, but the RS485 communication reliability, bit error rate is very small, even if the parity check error code, just repeat once again is enough.

(3) Timeout control: the PC machine start the timeout control when it is waiting for the reply. If within the specified time has not received any response information, then repeat the command frame.

(4) Re sending control: if PC receives retransmission reply frame, receiving data is incorrect or no answer in time, repeat the command frame maximum retransmission 3 times [8-9].

\section{3 system communication process}

The main communication flow of intelligent initiation system is shown in figure 6.

1) System initialization: the power is applied to the bus controller, so that the the power supply and the initialization operation is completed.

2) System test:

- bus controller receiving from PC system self checking instructions, closed detection energy switch and forwarding self checking instructions broadcast to all detonator;

- When state detection of each detonator is completed, self results will returned to the bus controller in different time;

- The bus controller then uploaded to the PC machine, PC machine system self-test results display in the user interface.

3) Charging: after the system self-test is normal, PC machine will send charging instructions.

- Bus controller receives a charging instruction from PC, turn off the charging energy switch,and the charging instruction is transmitted to the initiator of the address;

- When the charge is completed, the initiator will return the charge state in time (single site mode) or the time (broadcast mode) to the bus controller;

- The bus controller then uploaded to the PC machine, PC machine will be charging status display in the user interface.

4) ignition: charging is completed, the PC machine will send fire instructions. According to the different firing instructions, it can achieve synchronous firing, asynchronous firing and delayed firing, etc.

- The bus controller will be forwarded to the detonator ignition instruction address match;

- When the time is up,automatic ignition detonator;

- If not normal initiation, initiation device closes automatically discharging switch, the excess energy is withdrawn to prevent the detonator charged, which can increase the security of the system. 


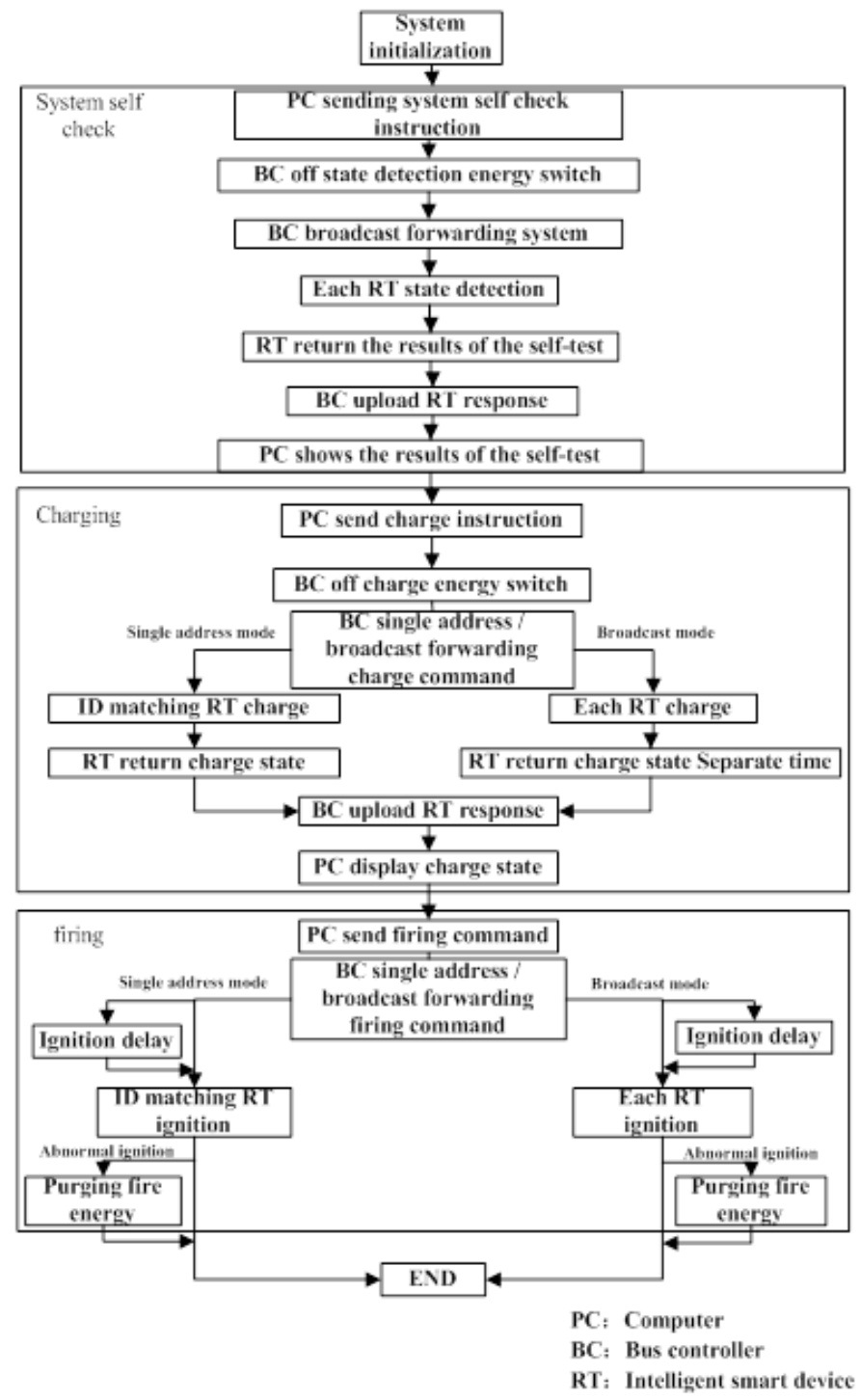

Fig. 6. Communication flow chart of intelligent initiating system.

\section{Summary}

In this paper, a communication protocol based on RS485 bus is designed, which can realize the accurate and reliable communication between the bus controller and multiple intelligent initiating devices, in order to realize intelligent initiating system more precise ignition in the environment where the interference is serious.

In addition to the use the RS485 bus which have strong anti-interference ability, open good, fault-tolerant ability of communication protocol is also the key to ensure data efficient transmission.RS485 bus protocol designed in this paper have a reasonable Frame structure, perfect error control mechanism. The bus controller is controlled by a command / response mode to the control all the initiating devices in single address or broadcast way.In this way, the reliable communication of the intelligent initiating system is ensured in the physical layer and the data link layer. 


\section{References}

1. Craig J. Boucher and David B. Novotney Ensign-Bickford Aerospace \& Defense Company Simsbury. "Connecticut Performance evaluation of an addressable integrated ordnance system", AIAA 2001-3636.

2. David B. Novotney and Andrew Kochanek, Ensign-Bickford Aerospace \& Defense Company, Simsbury, CT, 06070, “Intelligent Initiation Systems for Divert and Attitude Control Applications”, AIAA 2005-4502.

3. United States Patent, US 2004/0020394 A1, “ORDNANCE FIRING SYSTEM” , Feb. 5, 2004.

4. United States Patent, US 2005/0150998 A1, “ORDNANCE SYSTEM WITH COMMON BUS, METHOD OF OPERATION AND AEROSPACE VEHICLE INCLUDING SAME” , Jul. 14, 2005.

5. Yang Jin-yan, Zheng Ying-qiang, Zhang Zhen-ren. 8051 single chip microcomputer data transmission interface technology and application examples. People's Posts and Telecommunications Press, 2005.

6. Wang Bao-li, Guo Bao-yi. The design and implementation of RS485 based electronic cap initiation network and communication protocol. Journal of Shaanxi University of Technology, 2010, 26(1):20-23.

7. Han Ke-hua, Ren Xi, Li Li-ming. Application design of RS485 bus in the long distance multi point ignition system. New special device application, 2008, 10(9): 31-34.

8. Liu Ting. Design and verification of a high performance ordnance initiation system bus. Shanghai Jiao Tong University, 2007. 